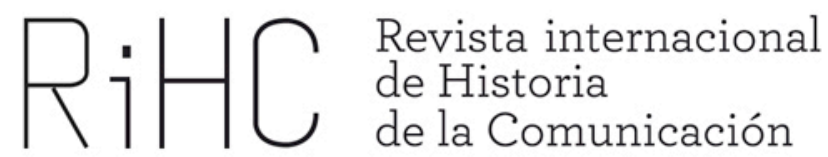

\title{
COMUNICACIÓN, POLÍTICA Y DEMOCRACIA EN ANTONIO PASQUALI
}

DOI: http://dx.doi.org/10.12795/RiHC.2017.i09.03

Roberto Emiliano Sánchez Narvarte ${ }^{1}$

Universidad Nacional de La Plata

emiliano.sanchez@perio.unlp.edu.ar

Recibido: 15-6-2017

Aceptado: 8-11-2017

\section{Resumen:}

El objetivo de este artículo es realizar una sistematización del pensamiento de Antonio Pasquali a lo largo de su trayectoria intelectual. Pasquali es un protagonista central del desarrollo de los estudios en comunicación latinoamericanos. Sus preocupaciones sobre la relación entre medios masivos, política y democracia en Venezuela fundaron una línea de investigación que se ocupó de pensar desde finales de los años sesenta hasta la actualidad, las políticas de comunicación.

Palabras clave: Antonio Pasquali, Democracia, Política, Medios Masivos

\footnotetext{
1 Roberto Emiliano Sánchez Narvarte es profesor Adjunto de la Cátedra III “Modernidades, Medios y Poder", Facultad de Periodismo y Comunicación Social (FPyCS), Universidad Nacional de La Plata (UNLP), y miembro del Instituto de Estudios Comunicacionales en Medios, Cultura y Poder (FPyCS-UNLP), además de becario de Investigación Doctoral del Consejo Nacional de Investigaciones Científicas y Técnica.
} 


\begin{abstract}
:
This article's purpose is to perform a systematization of Antonio Pasquali's thoughts along his intellectual trajectory. Pasqualis is a central character in the Latin-American communication studies development. His concerns about the relationship between mass media, politics and democracy in Venezuela founded a particular line of investigation which managed to think from the late's 60's until our days, the communicationpolitics.
\end{abstract}

Keywords: Antonio Pasquali, Democracy, Politics, Massmedias

\title{
1 Introducción
}

Italiano de nacimiento y venezolano por adopción, Antonio Pasquali (1929), formado en Filosofía y Letras en la Universidad Central de Venezuela (UCV), tuvo un temprano encuentro a principios de la década del cincuenta con círculos intelectuales que empezaron a problematizar los modos de producción cinematográfica. A partir de ello, de esas discusiones en la universidad y en redes culturales caraqueñas, es que al viajar a Francia a doctorarse en Filosofía, decidió realizar un curso de Filmología en el Institut de Filmologie de L'Université de Paris. Esta participación en distintas instituciones y organizaciones culturales parisinas, fue un pasaje formativo importante en la configuración de su inquietud por la pregunta en torno a los medios de comunicación y la cultura de masas.

Por otro lado, y en un plano regional, es hacia los años sesenta que comenzaron a surgir las primeras reflexiones, producciones y sistematizaciones en torno a la "problemática comunicacional" en América Latina (Munizaga y Rivera, 1983; Anzola y Cooper, 1985; Marques de Melo, 1987; Martín-Barbero, 1987; Fuentes Navarro, 1991 y 1992; Rivera, 1997; Saintout, 2003; Zarowsky, 2013).

Producto de sus primeras problematizaciones hacia aquellos años, es que Antonio Pasquali ha sido considerado como uno de los "padres fundadores" en los estudios comunicacionales en la región (Fuentes Navarro, 1991: 11).

A propósito de lo anterior, en su itinerario se articula su rol como académico, intelectual y "experto" en políticas de comunicación. Primero en el Ministerio de Educación del Estado Venezolano y, años después, del Consejo Nacional de Cultura. Hacia finales de los setenta, en 1978, ingresó como Subdirector adjunto en Comunicación y Cultura en la UNESCO, donde se jubila en 1989.

Desde su entrada en la universidad en 1958, se incorporó como profesor de Ética en la Escuela de Filosofía de la Facultad de Humanidades y Educación (UCV) y luego como 
docente del curso "Periodismo radial, cinematográfico y televisivo"2 en la Escuela de Periodismo (UCV).

En el plano de la investigación, entre 1974 y 1978 dirigió en Caracas el Instituto de Investigación de la Comunicación (ININCO), primer instituto de estas características en América Latina. Desde su carácter de experto, condujo en 1975 el "Proyecto RATELVE", un antecedente ineludible en América Latina para construir políticas comunicacionales, al problematizar la concentración mediática y proponer reformas en la legislación en pos de generar mayores condiciones de acceso y participación ciudadana en los medios masivos.

Desde el mismo ININCO, en 1978, fue uno de los profesores encargados de diseñar la primera Maestría Latinoamericana en Políticas de Comunicación, aprobada en 1980 (Pineda de Alcázar, 2014: 22).

Por otro lado, también hacia 1978 y junto a referentes de los estudios de comunicación formó la primera red latinoamericana de investigadores, la Asociación Latinoamericana de Investigadores de la Comunicación (ALAIC), y a finales de 1980, en el marco de una articulación con investigadores e intelectuales, fundó la Unión Latinoamericana y del Caribe de Radiodifusión (ULCRA), espacio que se propuso discutir críticamente el avance de los procesos de privatización de los medios masivos en la región (Pasquali y Vargas Araya, 1990).

\section{Objetivo y Metodología}

Este artículo es una sistematización parcial de la producción intelectual de Antonio Pasquali. Desde sus primeros trabajos de principios de los años sesenta hasta aquellos situados a finales de la década de los ochenta.

Puntualmente, se propone identificar y abordar críticamente los intentos del teórico venezolano de analizar y explicar los procesos comunicacionales, como así también, su participación y aportes en los procesos de elaboración de políticas y regulaciones para el sector mediático.

La estructura elegida para la exposición se compone de tres partes: en la primera se pretenderá dar cuenta del desplazamiento teórico e intelectual, su pasaje de la filosofía a la comunicación, a través de su acercamiento a los estudios filmológicos. Luego trataremos de indicar los principales nudos teóricos propuestos por el autor en

\footnotetext{
${ }^{2}$ Posteriormente, este curso fue incorporado en la currícula de la Escuela de Periodismo y pasó a tener rango de cátedra y a denominarse "Información Audiovisual" (Safar, 1978). Su fundador y primer titular fue el propio Pasquali.
} 
torno a la comunicación y a la cultura, y de qué modo intentó abordar a los medios masivos como problema político y económico. Por otro lado, haremos un breve comentario sobre su propuesta de una "moral de la comunicación".

En la segunda parte se trabajará en torno a las proposiciones que realizó en materia de políticas para el sector de la cultura y la comunicación, no sólo pensando en Venezuela, sino también cómo emergió la posibilidad de construir un proyecto a escala regional.

Y en la tercera parte, a modo de cierre, se realizarán una serie de interrogantes que permitan seguir problematizando el trabajo intelectual de Antonio Pasquali.

Para el abordaje se utilizará una perspectiva teórico-metodológica situada en el cruce de la historia de las ideas e historia intelectual. Situar la indagación en el espacio fronterizo de estos dos campos, permite, desde de la historia de las ideas, "comprender las creencias e ideas del pasado" a partir de indagar discursos, conceptos, representaciones de los intelectuales en torno a una época y problemas específicos (Terán, 2008: 11). De todos modos, trata de situarse en el plano de la producción conceptual sin perder de vista que las ideas, siendo productos de las relaciones sociales, surgen bajo condiciones histórico-materiales que llevan a reinscribirlas en "sus múltiples tensiones, ambivalencias y su pluralidad de situaciones" (Dosse, 2007: 36). Se trata de indagar, entonces, el "trabajo del pensamiento en el seno de las experiencias históricas" (Altamirano, 2005: 10).

\section{Breve estado de la cuestión}

En lo que se refiere puntualmente a los estudios acerca de la obra de Pasquali, nos encontramos con una serie investigaciones ineludibles e insoslayables acerca de su obra.

En principio, los ensayos que han indagado más integralmente las investigaciones de Antonio Pasquali son el trabajo conjunto de David De los Reyes y José Fidel Torres Duarte (2009) y la compilación de artículos realizada por Marcelino Bisbal y Andrés Cañizález (2014), ambas obras editadas por la Universidad Católica Andrés Bello (UCAB).

En el primero de estos trabajos, De los Reyes circunscribe su análisis en algunos desarrollos conceptuales de Pasquali, particularmente el de comunicación, y Torres Duarte hace hincapié, desde el campo filosófico, en la ética de la comunicación propuesta por el investigador venezolano. 
Con respecto al trabajo de Marcelino Bisbal y Andrés Cañizález (2014), si bien el punto de partida es la conmemoración de los cincuenta años de la publicación de Comunicación y cultura de masas (1964), a lo largo del mismo se expresan diversas miradas, perspectivas y abordajes en torno a distintos aspectos de su producción intelectual.

En lo que se refiere a estudios de carácter sistematizador de problemáticas, objetos y metodologías en general en América Latina y en particular en Venezuela, se encuentran ensayos que han acentuado en el modo en que Pasquali trazó una distinción conceptual entre información y comunicación (Mujica, 1973 y 2010 [1967]). Dicha conceptualización, sostienen algunos investigadores, le permitió pensar críticamente las relaciones de poder entre los medios masivos de comunicación y la sociedad (Aguirre y Bisbal, 1981; Aguirre, 1996; Cisneros, 2002; Beltrán; 2006; Silva, 2011 [1970]; Delgado Flores, 2014).

Por otro lado, Pasquali ha sido considerado como el "introductor" de la Escuela de Frankfurt en América Latina a partir de Comunicación y cultura de masas. En este sentido, su análisis crítico de los medios de comunicación, como también el rol de la cultura en los procesos de "mistificación" de las masas, ha sido entendido como un abordaje crítico que se inscribió en la matriz teórica proveniente de la Escuela de Frankfurt (Saintout y Díaz Larrañaga, 2003; Torres y De los Reyes, 2009; Varela, 2010; Holhfeldt, 2010; Pineda de Alcázar, 2010 y 2014; Cañizález, 2014).

En lo referente a sus elaboraciones en torno a la necesidad de formular políticas para el sector de las comunicaciones, se encuentran una serie de importantes trabajos. Por un lado, desde una perspectiva más bien histórica, se destacan los análisis de Oswaldo Capriles (1976, 1979, 1980 y 1996) y Peter Schenkel (1981), que dan cuenta de las condiciones socio-políticas en las que se desarrolló el Proyecto RATELVE como así también analizan el marco general de las Políticas Nacionales de Comunicación. Otro aporte importante es el de Margarita Graziano ${ }^{3}$, que destaca la originalidad del carácter socio-político de los estudios de Pasquali y sitúa a Comunicación y cultura de masas desde una perspectiva diferente a otras investigaciones, ya que lo entiende como un ensayo que pretendió "desmontar la estructura del emisor" y lo consideró como un antecedente ineludible de las posteriormente denominadas Políticas Nacionales de Comunicación (1988: 2; 1997: 3).

\footnotetext{
${ }^{3}$ Tanto Oswaldo Capriles como Margarita Graziano fueron miembros activos del ININCO en Venezuela. Capriles, que provenía del Derecho, se incorporó tempranamente a las investigaciones acerca de la comunicación y los medios. Fue director del ININCO entre 1980-1981 y 1982-1986. Graziano, en cambio, producto de su exilio durante la última dictadura militar argentina (1976-1983), se sumó al equipo a mediados de los setenta y participó en diferentes investigaciones, entre otros, el Estudio de Factibilidad para el establecimiento de un sistema de radiodifusión en Ciudad Guayana, coordinado por el propio Pasquali (1977, Ediciones ININCO). Graziano volvió a la Argentina tras el retorno de la democracia.
} 
En una línea similar a la de Graziano, en la que se entiende como central la articulación comunicación y política pero al mismo tiempo se amplía hacia reconocer su aporte al estudio de las industrias culturales latinoamericanas, se encuentran los trabajos de Martin Becerra (s/f) y Becerra y Guillermo Mastrini (2006). Por otro lado, también se destaca el artículo de Mastrini (2011) en el cual pone de relieve el aporte de Pasquali para pensar los servicios públicos de radiodifusión, como así también su tarea en la formación de la Unión Latinoamericana y del Caribe de Radiodifusión (ULCRA).

\subsection{Dimensiones teóricas y epistemológicas}

\subsubsection{Los medios masivos como problema de las ciencias sociales}

Como otros compañeros de su generación4, en 1955 y tras obtener la licenciatura en filosofía, Pasquali viajó a Europa a doctorarse. Producto de sus buenas calificaciones obtuvo una beca de la UCV y de ese modo pudo financiar su estancia doctoral en Francia.

Allí convergieron dos dimensiones diferentes de su formación intelectual: por un lado la estrictamente filosófica y por otro su disposición a la temática cinematográfica. En cuanto a lo filosófico, Pasquali se doctoró con una tesis sobre el problema conceptual de la libertad en los pensamientos de los filósofos Charles Renouvier y Henri Bergson. Tal como lo certifica su título doctoral, el 2 de julio de 1957 presentó la tesis Renouvier et Bergson: le probléme de la liberté y el jurado compuesto por Jean Wahl, Paul Ricœur y Vladimir Jankélévitch lo evaluó con "Muy Honorable" - máxima mención-. $Y$ en cuanto a lo cinematográfico5, se anotó en unos cursos bianuales -por fuera de la currícula del doctorado- que se dictaban en el Instituto de Filmología perteneciente al Centre Audio-visual de Saint Cloud de la Facultad de Letras, dirigido por Gilbert CohenSéat. En estos cursos se discutía y conceptualizaba sobre el fenómeno audiovisual; la televisión, la cinematografía y la radio.

\footnotetext{
${ }^{4}$ Por mencionar solo algunos: Federico Riu, tras obtener una beca por sus calificaciones, viajó a la Universidad de Friburgo y Juan Nuño viajó primero a Cambridge y luego a la Sorbona.

${ }^{5}$ Tras cursar la materia Historia del Arte en la UCV, Pasquali fue convocado por su titular Gastón Diehl para que se incorpore como ayudante a la cátedra en 1951. A través de Diehl, Pasquali ingresó a un universo cultural hasta el momento inexplorado. Diehl, historiador y crítico de arte de origen francés,

fundó el Círculo Universitario de Cine. El Círculo pretendió divulgar obras fílmicas, establecer vínculos especializados entre Europa y América, organizar ciclos de charlas y conferencias relativas a la historia, desarrollo y actualidad del cine. "El comité fundador estuvo integrado por el profesor Diehl y, entre otros, el estudiante Antonio Pasquali" (Álvarez y Rivera, 2011: 22-23).
} 
Según el certificado de titulación otorgado por el Instituto de Filmología, el 29 de mayo de 1957, Pasquali presentó una tesis titulada Notes pour une théorie de l'objet filmique y tuvo como profesores a Jean Wahl, Henri Dieuzeide y Edgar Morin, entre otros.

Luego de doctorarse en Francia en 1957, volvió a Venezuela y se incorporó al plantel docente de la UCV y sus primeras elaboraciones indicaban el desplazamiento de sus preocupaciones teóricas hacia las problemáticas de la comunicación y la cultura6. Como titular de la cátedra "Información Audiovisual", compiló un trabajo titulado Antología de textos para la cátedra de Información Audiovisual (UCV, 1960). Esta obra presentó como rasgo novedoso la preocupación de Pasquali por situar los estudios sobre la producción audiovisual y en general sobre los medios masivos, al interior del campo de las ciencias sociales (Pasquali, 1960).

En dicho texto se planteaba que los "nuevos mass media audiovisuales", como el cine y la televisión, habrían rebasado el marco de análisis lingüístico incorporando un componente de repercusiones problemáticas: la perspectiva social. La reflexión que emergía, según Pasquali, era que a los mass media había que pensarlos articuladamente desde dos dimensiones: una de orden semántico y estético, y otra como cuestión y praxis de las ciencias sociales (Pasquali, 1960: 8). Es decir, debían ser repensados como un problema analítico y como escenario de intervención del cientista social.

En este sentido, al inscribir la "cuestión de los mass-media" desde un enfoque social, planteaba que era necesario indagar las configuraciones sociales de los medios, es decir, entenderlos dialécticamente en tanto productos de las relaciones humanas, como dispositivos que relacionaban a hombres y grupos, y también en cuanto a las reconversiones o transformaciones culturales que pudieran llegar a producir los fenómenos audiovisuales.

Con respecto a la dimensión lingüística de la producción audiovisual, Pasquali consideraba que no era una simple forma de expresión, sino más bien un modo de comprensión del mundo (Pasquali, 1960: 59). Porque tanto en la expresión lingüística como en la iconográfica, se producían dos momentos analíticos en los que se objetivaba la realidad: un momento de comprensión, es decir, de representarse mediante el lenguaje audiovisual la realidad, y un segundo momento de fenomenización de lo comprendido en términos de lenguaje audiovisual (Pasquali, 1960: 60). Es decir, de construcción de un producto audiovisual que diera cuenta de la realidad que se pretendía comunicar.

\footnotetext{
${ }^{6}$ Enunciaremos brevemente que sus primeras participaciones en los debates intelectuales en torno a los medios, la cultura y la educación, se condensan en sus artículos "Deberes de la crítica cinematográfica" (1957), publicado en la Revista Nacional de Cultura, "Los intelectuales y el lenguaje audiovisual" (1958), en la revista Cultura Universitaria, y un largo ensayo titulado "Cine y Pedagogía" (1958), publicado originalmente en el diario El Universal, de Caracas. Estos artículos integraron, posteriormente, la Antología (1960).
} 
El estudio de los mass media permitía, según el autor, indagar las configuraciones sociológicas ubicando el problema comunicacional en la realidad social en la que emergía. Esto implicaba situarlo en un análisis mayor de las estructuras sociales y entender sus conexiones con los procesos sociales (Pasquali, 1960: 202). Al comprender que los medios de comunicación eran producto de las relaciones sociales, consideró que lo que allí se exponía era una visión subjetiva del mundo. Y que por lo tanto la producción audiovisual se convertía en un gran instrumento para la distribución de las ideas. Toda producción, en este sentido, era el medio ideal para vehiculizar un mensaje, una concepción del mundo (Pasquali, 1960: 250). Es por ello que la producción audiovisual debía ser pensada no simplemente desde el punto de vista estético, sino que se debía construir un marco de análisis que multiplicara las perspectivas analíticas y permitiera una comprensión total de los fenómenos audiovisuales. Es decir, analizar las dimensiones estéticas, éticas y político-sociales que se desprendían de él.

\subsubsection{Hacia una sociología de la producción cultural y comunicacional}

Desde sus primeras elaboraciones Pasquali comprendió a la comunicación y sus medios materiales en relación con las formas de organización social. Es decir, producto de su lectura dialéctica de lo social, entendió a los procesos comunicacionales en relación con las configuraciones sociales y políticas.

Si bien consideramos que realizó un planteamiento sociológico y materialista de la comunicación, el mismo Pasquali se encargó de afirmar que no se trataba de entender a la comunicación como reflejo o producto mecánico de las estructuras sociales. En todo caso, su inscripción sociológica y materialista se entiende a partir de considerar sus trabajos como un intento de construir un método cognitivo que permitiera dar cuenta del carácter humano, histórico y dialéctico de la comunicación. Planteamos que es sociológico porque situó los procesos de producción cultural y comunicacional en el marco del entramado de relaciones sociales que lo configuraban de una manera específica en una sociedad particular. Esto implicó, en su abordaje, pensar dichos procesos productivos sujetos a ciertas condiciones sociales e históricas, es decir, a sus condiciones materiales.

En este sentido, la primera consideración en torno a cómo Antonio Pasquali entendió a la comunicación, es que realizó una serie de inversiones teóricas: en primer lugar, comprendió a la comunicación no como un efecto secundario de la realidad, sino que intentó dar cuenta que la sociedad no era solamente una estructura de elementos políticos y económicos, sino también un proceso por medio del cual los sujetos se vinculaban, aprendían y comunicaban cosas: la comunicación adquiría una dimensión constitutiva de lo social en cuanto proceso necesario de sociabilidad. En segundo lugar, y en relación con lo anterior, es que partió teóricamente de entender que no era 
posible que se estructuraran determinadas relaciones y procesos sociales sin que haya una instancia de "aceptación" a través de la comunicación. O dicho de otro modo, pensar las relaciones de poder considerando una doble dominación económica y simbólica: un pueblo explotado económicamente que es hegemonizado mediante los productos culturales. Es decir, no podían producirse instancias de dominación si en simultáneo no se generaban las condiciones para que ciertos sectores de la sociedad adoptaran pautas y estrategias de los sectores dominantes, mediante este particular mecanismo que alienaba culturalmente (Pasquali, 1964b: 189).

Esta perspectiva de trabajo las elaboró centralmente en Comunicación y cultura de masas (1964a), El aparato singular (1967) y en Sociologia e Comunicação (1973), si bien hemos relevado trabajos previos en los que venía desarrollando estas problematizaciones ${ }^{7}$.

En estos primeros trabajos y en parte en el posterior Comprender la comunicación (1978), Pasquali reelaboró críticamente, a la luz de diversas matrices teóricas, una serie de conceptos como el de "comunicación", "información", "medios de comunicación de masas", "cultura de masas" y "medios de información", entre otros (Pasquali, 1964a).

Entendemos que dichos conceptos fueron aplicados en su producción como "ideas regulativas" (Kant, [1781] 1984) que le permitieron realizar una doble operación crítica: por un lado, en un sentido más estrechamente kantiano, le proporcionaban un horizonte conceptual a su comprensión del proceso social, un marco crítico desde el cual observar la realidad. Pero no deben entenderse como axiomas para pensar la realidad comunicacional, sino más bien como problemas conceptuales para abordarla y comprenderla (Kant, [1781]1984: 362). Por otro lado, dichos conceptos emergían como herramientas críticas para desmitificar y denunciar aquello que se proponía estudiar. Es decir, tales conceptos le permitían fundar la crítica, sin por ello cosificarlos y/o convertirlos en dimensiones absolutas o "puras" en cuanto a su aplicabilidad a los procesos sociales ${ }^{8}$.

Consideramos que partiendo de estas "ideas regulativas" podía tensionar conceptualmente aquello a lo que hacía referencia. En definitiva, era una operación que hacía entrar en tensión el pensamiento y la realidad y, en todo caso -como lo veremos posteriormente- era la práctica política la que podría generar una síntesis entre idea y realidad. Porque al mismo tiempo que criticaba la realidad a luz de

\footnotetext{
${ }^{7}$ En el XIII Congreso Internacional de Filosofía realizado en la Ciudad de México en 1963, presentó la ponencia "Sobre algunas implicaciones dialécticas entre 'información' y 'cultura de masas"” (mimeo), donde elaboró los conceptos centrales que se publicaron posteriormente en su obra de 1964a.

${ }^{8}$ Se podría agregar que esta particularidad en su producción intelectual la había desarrollado en su trabajo Fundamentos gnoseológicos para una ciencia de la moral (1963, EBUC). Este estudio fue una edición revisada de su tesis doctoral.
} 
aquellos conceptos, fue reelaborándolos a lo largo de su producción en función de las transformaciones sociales.

Es así que trazando estas ideas analizó el sistema de producción de bienes culturales como dimensión complementaria del fenómeno general de la producción de valores de cambio. Es decir, analizó de qué modo los productos culturales que se difundían como "la totalidad cultural", no eran otra cosa que una selección particular que operaba en los procesos de "alienación cultural" (Pasquali, 1964b: 189). En este sentido es que pensó a la producción cultural y comunicacional como problema político y económico. La cuestión central en torno a por qué se propuso analizar esta dimensión productiva, es que advertía que la producción y circulación cultural pasaba cada vez más intensamente por la mediación de los mass media, ocupando estos un rol estratégico en la circulación de los objetos culturales. Esto, además, conllevaba sostener $o$ al menos suponer a título de hipótesis, que se desarrollaba sistemáticamente una política o una doctrina de acción por parte de las empresas mediáticas que se definía a partir de considerar a la cultura como una mercancía sometida a leyes particulares (Pasquali, 1964a: 62).

Para dar cuenta de ello, analizó el "cuadro sociocultural" venezolano a través del modo de producción televisivo, el cinematográfico y el radiofónico, para así trazar las líneas directrices de esa cultura que circulaba por los medios. Esto le permitía visibilizar los mecanismos de dirigismo y dependencia cultural. Si por un lado, la cultura que circulaba era programada por grupos con intereses particulares, por otro, estos grupos estaban sujetos en términos de capitales e ideológicos al dominio norteamericano que controlaba el contenido de la programación con la pauta publicitaria (Pasquali, 1964: 70).

Para dar cuenta de la acción sistemática de los mismos, reconstruyó el entramado y las alianzas entre el sector empresarial y el político. En este sentido, es que entendió que la difusión de los productos culturales debía ser pensada desde el tejido económico que la condicionaba. Por ello sostenía que las pautas programáticas de los medios masivos estaban subordinadas a los requerimientos ideológicos del mensaje comercial, y que la distribución de los medios se restringía a las áreas que a las grandes empresas y agencias $^{9}$ les resultaba más explotable comercialmente. Esto generaba, en consecuencia, profundas desigualdades entre distintas zonas geográficas ya que algunas, al no ser rentables en términos de densidad demográfica, no accedían a los servicios de radiodifusión (Pasquali, 1964a: 108).

\footnotetext{
${ }^{9}$ Hacía referencia a la alianza que se producía entre la Asociación Interamericana de Radiodifusión (AIR), la Sociedad Interamericana de Prensa (SIP), con las empresas y organismos locales como la Cámara Venezolana de la Industria de la Radio y Televisión (CVIRT), la Federación Venezolana de Agencias Publicitarias (FEVAP) y el Consejo Venezolano de la Publicidad. Según Pasquali, dicha alianza terminaba operando como un frente organizado que controlaba, oligopólicamente, la producción y circulación de los productos culturales, al amparo del Estado nacional.
} 
Y como una instancia secundaria, planteaba la necesidad de pensar la dimensión ideológica de los productos culturales, considerando que si estaban sujetos a la "razón comercial" (Pasquali, 1967: 61), también debían ser funcionales en términos de qué concepción del mundo, estilos de consumo y de vida expresaban tales objetos que producía la industria.

\subsubsection{La relación comunicación y cultura. La articulación es la economía política.}

Pasquali sostuvo que sólo se podían comprender las violentas reacciones de los poderes centrales a las conferencias intergubernamentales de políticas de comunicación a mediados de los setenta10, si se observaba el desplazamiento del nudo "poder-dependencia" hacia los sectores de la comunicación y la información, considerados al interior de los sectores "terciarios" y "cuaternarios" de la economía. El problema de la dependencia había emergido en sus primeras obras entendido como un proceso político y económico que estructuraba las sociedades latinoamericanas (Pasquali, 1964, 1967 y 1973). Por ello es que en la dimensión de la producción cultural-comunicacional, el subdesarrollo en América Latina, era producto necesario de dicha relación de dependencia. Esto lo visualizaba en cuanto relevaba la fuerte presencia de las empresas norteamericanas en la producción de noticias, de programas de radio y televisión y en los films que circulaban en la industria cinematográfica venezolana, en relación con la casi inexistente producción y circulación cultural nacional y latinoamericana.

De hecho, esta preocupación iría in crescendo en su producción. En los años setenta y ochenta, lo económico va a ir adquiriendo mayor presencia en sus elaboraciones. De allí planteó que la dimensión que anudaba a la cultura y la comunicación era la economía. En este sentido afirmó que en la comunicación se anudaba el poder tecnológico, científico y político, reconfigurándose allí el nuevo escenario de la "dialéctica poder-dependencia" (Pasquali, 1978: 161).

La conceptualización que realizó Pasquali fue la siguiente: lo comunicacional era una dimensión que atravesaba horizontalmente todas las ramas de la vida productiva (Pasquali, [1986b] 1991: 168). A propósito de ello, consideró que la densidad de la comunicación iba reconfigurándose e intensificándose de sector a sector. Es decir, si en el sector primario, lo comunicacional podía emerger en cuanto se realizaban acuerdos entre vendedores y productores de materias primas; si en el secundario adquiría una característica fuertemente instrumental, en tanto publicidad de los

\footnotetext{
${ }^{10} \mathrm{El}$ investigador Oswaldo Capriles ha estudiado en profundidad los debates que se dieron al respecto en Venezuela y en la región. Para ello, recomendamos leer sus trabajos El estado y los medios de comunicación en Venezuela (1976) y Poder político y comunicación (1996).
} 
productos manufacturados, en los sectores terciarios y cuaternarios, lo comunicacional alcanzaba un status profundamente diferente: en dichos sectores se condensaba lo comunicacional y la mercancía de forma indisociable. Es decir, la comunicación y la cultura eran, en simultáneo, dimensiones productivas y productos de la actividad terciaria y cuaternaria de la economía.

El problema, entonces, se daba en la reconfiguración de la industria cultural: la comunicación en tanto dimensión transversal del sistema productivo, se volvía imperceptible y omnipresente. En dicho escenario, entonces, lo comunicacional y cultural se volvía un recurso básico para gestionar y producir y también como materia prima para la obtención de plusvalía. Por ello planteó que en los flujos de información se consolidaban las desigualdades, un problema que además de la dimensión productiva, se desplazaba hacia la circulación y el consumo de los productos culturales (Pasquali, [1986b] 1991: 168).

En este marco la industria cultural debía ser repensada en términos conceptuales y como escenario de intervención. En unas condiciones sociales e históricas en las que según Pasquali, toda cultura estaba atravesada por un proceso industrial, negar ese carácter, era asumir una posición reaccionaria y de renuncia al problema (Pasquali, [1986b] 1991: 169). Por el contrario, en el marco de la regionalidad continental, los países del sur de América debían pensar estrategias de producción de servicios y bienes culturales, rentables y exportables, frente a los flujos internacionales generando las condiciones para el desarrollo de las industrias culturales nacionales (Pasquali, [1986b] 1991: 168). Sostenía que, si bien era cierto que la industria había sido históricamente estructurada bajo las leyes del mercado, podía ser transformada políticamente para promover un proceso de democratización cultural (Pasquali, 1988: 215).

Por ello es que casi al mismo tiempo, y puntualmente en trabajos como De la marginalidad al rescate: los servicios públicos de radiodifusión en la América Latina (1990), La comunicación cercenada (1990), El orden reina (1991) y Memorias de un país en subasta I y II (1992 y 1994), sus análisis se empezaron a desplazar de la pregunta por la producción cultural en un sentido más estricto, es decir, en el marco de las industrias culturales, hacia las condiciones materiales de las telecomunicaciones.

Si bien se mantuvo la pregunta por el carácter político y económico de las comunicaciones, adquirió otra dimensión al ser pensadas como las redes de circulación de la economía, la cultura y el saber. La pregunta que se formuló en estos trabajos fue cuáles eran las condiciones de acceso y participación de la sociedad venezolana en la producción económica, cultural y educativa. Puntualmente consideró a las telecomunicaciones cómo dimensiones centrales en los procesos de democratización, modernización e integración regional (Pasquali, 1990); integración entendida como 
unión económica y social entre los Estados latinoamericanos. Para este análisis se ocupó del correo, el turismo, la producción editorial, la telefonía, la prensa, la radio y la televisión.

El objetivo de analizar las dimensiones antes indicadas, según Pasquali, se vinculaban con la idea de dar cuenta de los mecanismos que constituían el entramado cultural y que imposibilitaban tal integración.

De todos modos, Pasquali no intentaba reducir los procesos culturales a las lógicas económicas. Al respecto, sostuvo que la cultura no debía entenderse como una dimensión superestructural sino como componente de la estructura (Pasquali, 1990: 25): es decir, la formación y las prácticas culturales históricamente producidas se volvían, en términos políticos, instancias que dificultaban los proyectos de integración, porque los países de la región si bien establecían acuerdos comerciales no cosideraban el entramado cultural. La cultura también debía ser pensada no solo como producto sino a sí misma como modo de producción de ideas, saberes y prácticas que desafiaban los proyectos de integración económica (Pasquali, 1990: 25).

En estos trabajos Pasquali volvió a traer a la escena el concepto de dependencia. Era el carácter dependiente de la industria cultural y de las telecomunicaciones lo que produjo históricamente tales diferencias culturales al interior de América Latina. Pero era una dependencia transformada: el proceso de concentración horizontal y vertical de las comunicaciones en el marco de un proceso regional de privatización de los servicios públicos (Pasquali, 1991:10).

A propósito de esto, y como lo sostuvo en Bienvenido Global Village (1998), este proceso traía como consecuencia nuevas instancias de disputa en el marco de la hegemonía del mercado y empresarial en lo referente a la producción cultural e ideológica, reconfigurando asimismo, las relaciones de poder entre gobiernos y empresas mediáticas. Estas últimas habían adquirido mayor preponderancia y fuerza para presionar a los gobernantes y de ese modo construir una opinión pública que legitimara sus intereses y posiciones. Lo que se ponía en juego era el sentido mismo de la democracia debido a que las alianzas entre el sector empresarial y el político se anudaban con más fuerza.

Y otra dimensión central del problema era la nula participación de la población en términos de producción cultural y comunicacional. El acceso a la pluralidad de fuentes y la diversidad cultural eran restringidas producto de la hegemonía de la industria del entretenimiento, convirtiendo a la cultura en mercancía estandarizada y al ciudadano en consumidor y no en productor cultural (Pasquali, 1998: 238). 


\subsubsection{De la ética a la moral de la comunicación}

A partir de sus producciones de mediados de los setenta propuso lo que dio en llamar en principio una "ética de las comunicaciones" y posteriormente una "moral de las comunicaciones", las que consideró como lo que debería ser el objetivo final de una teoría general de la comunicación (Pasquali, 1978: 130).

Las desarrolló fundamentalmente en el artículo "Latin America: our image or theirs" (1975) y en sus libros Comprender la comunicación (1978), El orden reina (1991) y en Bienvenido Global Village (1998).

Entendemos que este fue un proyecto inconcluso porque han sido desarrollos fragmentarios, no sistemáticos ni continuados a lo largo del tiempo, pero que de igual modo, algunos de sus aspectos, aparecieron transversalmente en otras de sus obras e incluso permitirían relacionarlos con sus posicionamientos políticos e intelectuales en los distintos escenarios en los que intervino.

El papel que le otorgó a la "ética de las comunicaciones" fue el de elaborar abstracciones y formalizaciones conceptuales, derivadas de los análisis empíricos, para ofrecer principios que puedan ser aprovechables en las formulaciones de normas comunicacionales por parte de los legisladores y los políticos (Pasquali, 1978: 130). El punto de partida estratégico para ello, se inscribía en la definición misma del proceso comunicacional, la cual indicaría por defecto, una posición ideológicoconservadora de lo establecido o una posición utópica-renovadora del orden (Pasquali, 1975: 58). En la definición conceptual se visibilizaba el posicionamiento político frente a los procesos comunicacionales. La definición propuesta estableció una equivalencia entre la idea de "comunidad" (Kant, [1781] 1984: 146) con "comunicación". Esta operación le permitía a Pasquali plantear la necesidad de establecer como condición del proceso comunicacional, la reciprocidad entre los sujetos.

Como se sostuvo anteriormente, situarse aquí lo habilitaba a denunciar el carácter no recíproco de la comunicación en América Latina y, por lo tanto, su definición se proyectaba como lo que no se había cumplido aún, como una situación a la que se debía llegar, y que en todo caso era el proceso histórico social el que había impedido una auténtica relación comunicacional (Pasquali, 1978: 277).

El problema, según Pasquali, tenía dos dimensiones: una "natural", por las mismas dificultades técnicas que proponían los medios masivos para el desarrollo de la reciprocidad; y otra explícitamente política en cuanto a los problemas del control de la producción y circulación de los mensajes. Este primer momento de la "ética" se tornaba un discurso político en cuanto relacionaba la problemática con en el uso de los grandes medios de comunicación en tanto denunciaba que la imposibilidad del intercambio de la palabra, no debía justificarse por la dimensión técnica que restringía 
una auténtica comunicación, sino por el carácter político que organizaba y orientaba la unidireccionalidad de los medios masivos (Pasquali, 1975: 59).

Para revertir dicha situación, entonces, se debían aportar sistemas categoriales, criterios y principios que reorientaran la actividad concreta de sociólogos, políticos, comunicadores y legisladores en los centros de decisión nacionales e internacionales con capacidad y poder para generar cambios sociales (Pasquali, 1978: 145).

\subsubsection{Segundo momento: una "moral de la comunicación"}

Posteriormente, Pasquali se desplazó de la idea de formular una ética hacia una moral de la comunicación. Es decir, de la preocupación por crear sistematizaciones epistemológicas que orientaran la praxis en el campo de las comunicaciones, hacia la reflexión sobre una "nueva moral" 11 de la intersubjetividad, que reorganizara la acción comunicacional de modo tal que todos los ciudadanos pudieran expresarse y comunicarse por todos los medios (Pasquali, 1998: 20).

Las razones que daban sentido a este desplazamiento se encontraban, según Pasquali, en que se había producido una reconfiguración del orden informacional y comunicacional. Este había efectuado, bajo la "máscara de la pluralidad", procesos de desregulación en términos de políticas comunicacionales que lejos de generar mayores condiciones de acceso y participación, reconcentraban el sistema de producción cultural y comunicacional desmantelando los servicios públicos radiotelevisivos (Pasquali, 1998).

Según el teórico venezolano se habían alterado los mecanismos tradicionales del ágora, como espacio de discusión, debate y formación de la opinión pública, al acentuarse el carácter mediador de los medios masivos. La mediación entre realidad y ciudadanos que asumían unos medios masivos fuertemente concentrados y "mercado dependientes" cuestionaban el sentido mismo de la democracia (Pasquali, 1998: 23).

Por ello insistió en pensar el proceso comunicacional siempre anclado en la relación antropológica (Delgado-Flores, 2014: 95), lo que hacía repolitizar la técnica en cuanto insistía en que ésta debía entenderse a la luz de las relaciones histórico-sociales y políticas. En dicho sentido es que consideró que se debían devolver a todos los sujetos la posibilidad, entendida como un derecho humano, de comunicarse por todos los

\footnotetext{
${ }^{11}$ Esta problematización en torno a una "moral de la comunicación" entraba en dialogo con las elaboraciones del filósofo Jurgen Habermas en sus obras Teoría de la acción comunicativa (1981) y Cociencia moral y acción comunicativa (1983). Según el propio Pasquali, esos dos trabajos del pensador alemán constituían un punto de partida y todo un programa de investigación que debía profundizarse (Pasquali, 1998: 44).
} 
medios (Pasquali, 1998: 28). Si, como advertía en sus trabajos, el sector infocomunicacional se dirigía hacia procesos de profunda concentración, la posibilidad de comunicar se reducía cada vez a menos manos, tensionando la misma democracia.

Por ello es que proponía rehabilitar la relación intersubjetiva como condición necesaria de un proceso comunicacional democrático, de reconocimiento de demandas y necesidades del otro. Para esto se tornaba necesario formular estrategias para "liberar" la comunicación (Pasquali, 1998: 36). Una liberación, según Pasquali, del habla, del discurso y del diálogo en todas sus formas. Para ello había que construir procesos participativos concretos que reestructuraran y otorgaran representatividad a la población en los órganos directivos y de programación de los grandes servicios radiotelevisivos públicos, o en organismos públicos de supervisión y control (Pasquali, 1998: 37). La construcción, en definitiva, de nuevas políticas que buscaran transformar las prácticas culturales a partir de las cuáles la comunicación pueda volverse una categoría central de la nueva moralidad que permitiera nuevos modos de "estar juntos".

Siendo las comunicaciones uno de los sectores más conflictivos en términos de que históricamente se habían configurado sujeto a las reglas del mercado, se requerían nuevas regulaciones políticas y sociales para garantizar la libertad de comunicarse, reequilibrios, reciprocidades, derechos, deberes y responsabilidades (Pasquali, 1998: 38).

\section{Praxis política e intelectual}

\subsection{La elaboración de políticas culturales y comunicacionales.}

En las producciones intelectuales del teórico venezolano, se haya invariablemente, una recurrencia: su vocación por orientarse hacia lo concreto, de pensar cómo articular las elaboraciones teóricas con la intervención política. En este sentido, hacia finales de los setenta, advertía que "toda abstracción por la abstracción" que no estuviera destinada a "recuperar la dimensión antropológico, social y política del problema" comunicacional, resultaba sospechosa de favorecer a ciertos intereses ideológicos y económicos (Pasquali, 1978: 36).

Por ello es que no renunció a pensar a la comunicación y a los medios masivos como problema político. Consideramos que situar la cuestión allí, le permitió en términos teóricos, reorientar su pensamiento hacia la elaboración de estrategias y regulaciones 
que intentaran resolver los conflictos que se presentaban en el plano de la cultura y la comunicación.

En un primer momento, la propuesta de Pasquali adquirió un carácter "denuncista" del orden político y cultural y, por otra parte, intentó definir algunas líneas para solucionar el "problema de los medios" sin que sus ideas lograran materializarse en algún proyecto que pasara del carácter formalista o teórico.

Sus primeras formulaciones de políticas para el sector de la cultura y la comunicación, dijimos, fueron elaboradas en Comunicación y cultura de masas (1964a), y planteaba como alternativa al sistema establecido, para redireccionarlo, la intervención de los poderes públicos y la nacionalización de los servicios radioeléctricos de difusión (1964a: 243). Consideraba que el Estado era el único agente que podía usufructuarlo según políticas culturales dirigidas hacia el bien común. Esto implicaba elaborar una planificación cultural que permitiera garantizar los servicios de radio y televisión a todos los habitantes; reducir el impacto de las empresas comerciales publicitarias en la programación massmediática y la diversificación de la misma. Es decir, que la programación ya no estuviera sometida a la lógica comercial sino al interés común. De todos modos, la idea de "nacionalización" en Pasquali no implicaba la estatización del servicio radioeléctrico, es decir, que la producción del servicio fuera monopolizada por el Estado. Al contrario, matizó esa modalidad de intervención y planteó que era necesaria una centralización y planificación a escala nacional con una mayoría de capitales públicos y bajo el mandato de un instituto autónomo o consejo paraestatal y que si bien debía ser dependiente del Estado no así del gobierno (Pasquali, 1964b: 193).

De esta manera, consideramos, se inscribía en la tradición europea del servicio público, teniendo como máximo exponente a la British Broadcasting Corporation (BBC) de Londres, y como marco de referencia las orientaciones de la UNESCO. Las características que debía asumir el servicio público en cuanto a la gestión, eran que tenían que estar sometidos a la autoridad directa del Estado o bajo mandato de organismos públicos paraestatales.

Ese matiz mencionado más arriba, le bajaba el tono a la idea de "nacionalización", porque al pensar teóricamente un organismo paraestatal, y siguiendo la experiencia de la $\mathrm{BBC}$, se podía generar este organismo bajo una ley que garantizara su autonomía frente a controles de tipo político o comercial. En este sentido, el carácter radicalizado de la propuesta no estaba en la propuesta misma; básicamente, lo que Pasquali proponía era una política "progresista" que atendiera los requerimientos básicos de la colectividad, que los medios masivos sean refuncionalizados para "nivelar culturalmente" y llevar la idea de "progreso" a las regiones más "deprimidas" (Pasquali, 1964a: 98). 
En definitiva, la propuesta de Pasquali se orientaba hacia un sistema de producción cultural y comunicacional mixto, en tanto coexistieran dos tipos de propiedad en la que el Estado se reservaba una por derecho para su explotación y otra sea concesionada al sector privado. Si bien no podemos extendernos en este punto, consideramos, en todo caso, que no es la propuesta de Pasquali una idea radicalizada en términos de transformación total de modo de producción cultural; sino que fueron las fuerzas políticas y económicas concentradas de los medios masivos las que volvieron "radical" cualquier conceptualización que en cuanto a la producción en general, introdujera como variable una redimensión del rol del Estado y mayor participación del mismo.

Pero sería unos años después, a principios de la década del setenta, que la formulación de políticas para el sector cultural y comunicacional alcanzó, al menos en Venezuela, uno de los puntos más altos en términos de diagnóstico, sistematización y elaboración de regulaciones. Estas se condensaron en el trabajo colectivo del Comité de Radio y Televisión dirigido por Pasquali12, perteneciente al Consejo Nacional de Cultura. El Informe fue elaborado entre noviembre de 1974 y mayo de 1975, y fue editado en 1977 con el título Proyecto RATELVE. Diseño para una nueva política de radiodifusión del Estado venezolano.

Si bien no nos vamos a extender en la cuestión, es necesario no aislar este proyecto de las propuestas generales que se había fijado el gobierno de Carlos Andrés Pérez (19741978), representante de Acción Democrática. Mediante la Ley del Consejo Nacional de Cultura de agosto de 1975, el Poder Ejecutivo se había propuesto fijar "Ios principios rectores" de la política cultural del Estado, dirigida "fundamentalmente al estudio, planificación, coordinación y ejecución, en el campo de las humanidades, las artes y las ciencias sociales", principalmente en sus manifestaciones no escolarizadas. Para ello creó el Consejo Nacional de Cultura como Instituto Autónomo, adscrito a la Presidencia de la República, con personería jurídica y patrimonio propio. Lo relevante de esta Ley - y que generó posteriormente una enorme lucha entre los sectores gobernantes y los empresarios13- fue el artículo 4 que definía como "áreas prioritarias del campo de la cultura", todas aquellas manifestaciones que se expresaran "a través del mensaje cultural impreso, radio-eléctrico y cinematográfico" y, finalizaba, el Estado crearía y mantendría los "servicios que garanticen el disfrute de la cultura para todos los habitantes del país".

\footnotetext{
${ }^{12}$ Entre los redactores del Informe estuvieron Raúl Agudo Freites, Oswaldo Capriles, Héctor Mujica, Ovidio Pérez Morales, HelySanteliz y Francisco Tugues. Y como asesores externos participaron Luis Ramiro Beltrán y Elizabeth Fox de Cardona, entre otros.

${ }^{13}$ Si bien no podemos detenernos en este punto, diremos que Oswaldo Capriles ha reconstruido en profundidad los ataques que recibieron los redactores del Proyecto y, en general, las confrontaciones que se dieron entre las cámaras empresariales y el gobierno de Carlos Andrés Pérez. Ver en su trabajo Poder político y comunicación, el capítulo "El caso del Proyecto RATELVE en Venezuela" (1996: 75-121).
} 
Aun asumiendo que es un desarrollo muy parcial, diremos que el RATELVE tenía como objetivo general adoptar la forma de servicio público con la idea de promocionar, apoyar y proyectar la identidad nacional (AAVV, 1977: 285). Consideraba que sólo era posible lograr tal objetivo si el Estado asumía el control global de la radiodifusión y creaba una unidad político-administrativa que se encargara del sistema nacional de radiodifusión (AAVV, 1977: 286).

Dicha unidad político-administrativa, denominada "Consejo Nacional de Radiodifusión", debía convertirse en un organismo multisectorial que articulara a distintos actores sociales: directivos y docentes de distintos niveles educativos, gremios y sindicatos de trabajadores, asociaciones de autores y actores, las universidades, usuarios de la radiotelevisión y representantes del sector privado de las telecomunicaciones (AAVV, 1977: 338).

Inscribiéndose en las elaboraciones resultantes de los encuentros internacionales de la UNESCO, el proyecto asumía la idea de que la necesidad de independencia política, económica y cultural obligaban a los Estados de la región, a asumir "la dirección global de la política nacional de Comunicación Social hasta ahora ejercida parcialmente por la industria cultural privada" (AAVV, 1977: 48).

Partiendo de tal marco, sólo una planificación estatal del servicio de radiodifusión podría redireccionar a la industria cultural privada como un instrumento de progreso y cultura (AAVV, 1977: 50).

Si la producción cultural y comunicacional venezolana estaba constituida por un sistema competitivo según los cánones de la economía del mercado sin considerar las necesidades de los receptores, debía ser convertido en un sistema complementario en el que la radiodifusión se convirtiera en un servicio público y sus mensajes sean producidos y emitidos predominantemente por organismos estatales. El emisor público debía maximizar la cobertura a todos los ciudadanos y encargarse de diversificar la programación con el objetivo de ofrecer productos culturales que atiendan las necesidades y los deseos de la población (AAVV, 1977: 54).

En relación con el carácter complementario del sistema, el proyecto indicaba que proponían un régimen "mixto auténtico", en el que convivieran en relaciones de equilibrio respecto de la propiedad, el sector público y el privado. De este modo, el poder público debía dar cobertura territorial total y los usuarios podrían elegir entre una programación competitiva y complementaria. De todos modos el sector privado se debía ajustar las metas generales del servicio público (AAVV, 1977: 61-62). Es decir, la conducción política y cultural, aun sin estatizar el servicio, debía ser llevada adelante por el Consejo Nacional de Radiodifusión que planificaría en un marco de acuerdos y estrategias conjuntas, la programación y el desarrollo de medidas para el sector. 


\subsection{Las industrias culturales como estrategias de integración}

Desde finales de los setenta la Organización de las Naciones Unidas propuso, como mecanismo de colaboración sur/sur, la noción de Cooperación Técnica entre los Países en Desarrollo (CTPD), que buscaban, de alguna manera, intercambios de experiencias y asistencia técnica entre instituciones de los países en desarrollo. Teniendo este marco de cooperación regional, desde la UNESCO se fundó en 1985 la Unión Latinoamericana y del Caribe de Radiodifusión (ULCRA).

Pasquali fue uno de los promotores de la Unión al ser el Coordinador Regional de la UNESCO para América Latina y el Caribe, y como Director del Centro Regional de Educación Superior para América Latina y el Caribe (CRESALC) con sede en Caracas. Desde esta inscripción institucional, la ULCRA se gestó como un organismo internacional de consulta, cooperación y coordinación, que buscaba, según sus estatutos, promover el fortalecimiento de la identidad cultural y la integración audiovisual latinoamericana (Pasquali y Vargas, 1990: 131).

Básicamente, el objetivo de la institución era articular los servicios públicos de radiodifusión de la región. En este sentido, el servicio público se distinguía tajantemente del servicio gubernamental ya que necesariamente debía ser independiente respecto del poder económico y del político (Pasquali, [1986a] 1991: 153). Si bien su financiamiento provendría del erario público, este debía ser independiente, participativo y de alta calidad. En este sentido, ya explicitado el carácter de su independencia, se pretendía que distintas organizaciones pudieran ser parte de la gestión de las empresas de radiodifusión que aseguraran una participación concreta en la dirección de los mismos (Pasquali, [1986a] 1991: 154). Por otra parte, se planteaba que, si los fondos públicos provenían de la población, se debía construir y garantizar un vínculo entre los que lo financiaran y los que emitieran. Es decir, los servicios públicos debían cumplir con las demandas de la sociedad y no ajustar su programación a la lógica puramente comercial.

Otro de los objetivos de la ULCRA fue generar una red de producción, circulación y consumo audiovisual regional, que no sólo permitiera intercambios al interior del continente, sino también construir una plataforma a partir de la cual se exportaran los productos culturales hacia otras regiones del mundo (Pasquali y Vargas, 1990: 131) De todos modos, la organización advertía que el sur del continente estaba atravesado por un proceso de "fuerte atomización de los medios, desconexión y dependencia masiva de importación extra regionales" como característica central de los sistemas latinoamericanos de comunicación audiovisual (Pasquali y Vargas, 1990: 139). Para contrarrestar dicho estado de situación la ULCRA buscaba reducir la dependencia de la importación y generar una demanda de programas y servicios nacionales y regionales que consolidaran las industrias culturales endógenas. Ahora bien, esto no 
podría realizarse si, como se sostenía en el mismo documento, no se establecían políticas nacionales de información/comunicación que resguardaran la pluralidad cultural y política, reafirmando la soberanía nacional y movilizando la participación de la comunidad (Pasquali y Vargas, 1990: 152).

En el marco de la III Conferencia Latinoamericana sobre Servicios Públicos de Radiodifusión realizada en Quito en 1989, la Unión recibió el apoyo de los gobiernos de la región. Las declaraciones de dicha Conferencia entregaron una serie de postulados en torno a la necesidad de reconocer a la producción audiovisual como "instrumento para el desarrollo económico, la consolidación democrática, el progreso social y la educación popular" y la creación de un Espacio Audiovisual Latinoamericano que diera cuenta de las prácticas culturales de los pueblos miembros (citado en Pasquali y Vargas, 1990: 151).

De este modo, ese espacio audiovisual latinoamericano podía operar como frente regional que, desde una clave cultural, pudiera generar rentabilidad económica y proyectarse hacia el mercado internacional de manera articulada y programática. Un espacio que permitiría también revitalizar las producciones nacionales y generar coproducciones regionales. Se trataba de ingresar, como sostenía Pasquali, al "pool de productores y emisores de cultura", compartiendo riesgos y beneficios, de retener a los productores culturales locales, y "crear sólidos polos productivos" (Pasquali, 1988: 228).

\section{Conclusiones}

Como sostuvimos anteriormente, las elaboraciones teóricas de Antonio Pasquali siempre estuvieron orientadas hacia la práctica y la intervención política e intelectual. En este sentido, si bien consideramos que es una recurrencia importante en su trabajo, ha sido una constante que debe ser pensada desde sus transformaciones. Es decir, considerada su trayectoria y producción diacrónicamente, esa constante fue resignificándose, reelaborándose a partir de los procesos laterales, o sincrónicos. En este trabajo acentuamos más bien sus ideas de un modo lineal y no los debates y las discusiones teóricas en las que estas emergieron. Consideramos que es necesario trazar ese mapa de coordenadas para dar cuenta de las diversas tensiones que se anudan en los trabajos analizados.

En términos más concretos, en las mismas obras expuestas aquí identificamos debates que, entendemos, nos llevan a otras discusiones. Por un lado, y como sostiene Ángel Rama (1976: 29), en la década del sesenta en Venezuela se produjo una explosión de "energías y propósitos renovadores" que se diseminaron por distintos sectores de la producción cultural y artística.

Esa "izquierda cultural" venezolana, como la denominó Alfredo Chacón (1971), discutía y producía saberes en torno a la cultura, la política, el arte y los medios masivos, desde 
un tejido ideológico que articulaba proyectos de modernización y desarrollo del sistema cultural y educativo, como así también interpelados por los procesos políticos revolucionarios que se habían producido en Cuba.

Es decir, y ejemplificando lo anterior, ¿A partir de qué condiciones académicas, políticas y culturales, este joven filósofo se desplazó intelectualmente hacia las problematizaciones en torno a la comunicación y a la cultura? ¿de qué modo su participación en formaciones y organizaciones culturales -en el sentido de Raymond Williams (2009: 158)- caraqueñas de los años sesenta, como la revista Crítica Contemporánea o posteriormente Imagen o Cine al Día, operaron productivamente en la problematización de sus elaboraciones teóricas?

Con respecto a las condiciones de producción de sus textos de principios y mediados de los setenta, ¿en qué debates políticos e intelectuales emergió la problemática de la comunicación y los medios masivos en Venezuela? ¿Qué relaciones se pueden establecer con los debates en otras academias latinoamericanas? ¿Qué discusiones se planteaban entre las distintas facultades y redes intelectuales -como las nucledas en Orbita, en Video-Forum, en Comunicación y la revista ININCO entre mediados del setenta y principios del ochenta? ¿De qué modo se inscribió Pasquali en dicho proceso?

Sus producciones, consideramos, se dieron en un campo de fuerzas trazado por discusiones, organizaciones culturales e instituciones académicas. $Y$ que lejos de excluirse una a otra, en algunos momentos convergieron críticamente.

Como indicativo de ello, en diálogos mantenidos con el propio Antonio Pasquali ${ }^{14}$ y con el Arzobispo Ovidio Pérez Morales ${ }^{15}$, el primero Director del Proyecto RATELVE y el segundo uno de sus miembros redactores, sostuvieron que, en las reuniones para su elaboración, se dieron múltiples discusiones entre las diversas posiciones ideológicas que tenían los miembros de la comisión redactora. Vale recordar, que allí participaron representantes del gobierno, de las Fuerzas Armadas, profesionales e investigadores de la comunicación y de la Iglesia. Esto conllevaba a que se produjeran intensos debates entre posiciones divergentes -aunque no necesariamente antagónicas.

A modo de ejemplo, si se atiende a las propuestas del RATELVE, emerge esa diversidad. La propuesta, en definitiva, se propuso como un intento de redimensionar el rol del Estado para que asuma la dirección político-jurídica del sistema de producción cultural y comunicacional, como condición para la modernización y de desarrollo del sector. Pero al mismo tiempo, debía ser un desarrollo autónomo que permitiera, simultáneamente, romper los lazos de dependencia nacional.

\footnotetext{
${ }^{14}$ Entrevista concedida al autor, 20 de febrero de 2015, Caracas, Venezuela.

${ }^{15}$ Entrevista concedida al autor, 11 de marzo de 2016, Caracas, Venezuela.
} 
En ese sentido, situar el problema del monopolio de los medios de comunicación de masas en unas burguesías nacionales subordinadas al poder extranjero, no dejaba de ser una crítica radical de la reproducción del orden, y tal como observamos en las propuestas, que debía ser reformada políticamente.

Por otro lado, consideramos que es apropiado y necesario pensar cómo se fue articulando su particular formación filosófica con las tradiciones intelectuales europeas que se preguntaron por la filmología. Complementariamente, pensar sus diálogos y tensiones con la matriz funcionalista norteamericana dominante en las facultades de periodismo y comunicación venezolanas hacia finales de los años sesenta, cuando se inicia, según Jesús María Aguirre, el "lento proceso de ruptura" con esa perspectiva norteamericana (Aguirre, 1996: 43-49).

Al mismo tiempo, preguntarse por las condiciones en las que empezaron a circular obras y trabajos provenientes de Europa, de matriz marxista como los de algunos pensadores de la Escuela de Frankfurt, por ejemplo. Y en ese contexto, en todo caso, en qué Frankfurt abrevó Pasquali, y qué le permitió pensar en torno a las condiciones específicas de la región. Y además, los intercambios y diálogos con otros investigadores latinoamericanos que empezaron a circular por el sur del continente desde la década del setenta.

Creemos que algunas de estas preguntas nos conducen a indagar ya no solo el pensamiento de Antonio Pasquali, sino a articular sus problematizaciones teóricas con su itinerario intelectual, con las redes académicas y culturales que frecuentaba, y a reconstruir cuales fueron sus posicionamientos y preocupaciones frente a la emergencia de distintos movimientos políticos.

Por último, entendemos que lejos de ser un punto de llegada, las conceptualizaciones desarrolladas y las preguntas enunciadas, configuran una plataforma para seguir reflexionando sobre sus ideas y aportes a los estudios en comunicación y cultura latinoamericanos.

\section{Referencias bibliográficas}

AAVV, (1977): Proyecto RATELVE. Diseño para una nueva política de radiodifusión del Estado Venezolano, Venezuela, Librería SUMA.

AGUIRRE, J. M. y BISBAL, M. (1981): La ideología como mensaje y masaje, Caracas, Monte Ávila Editores.

AGUIRRE, Jesús María (1996): De la práctica periodística a la investigación comunicacional, Venezuela, Polar-UCAB. 
(2014): Vigencia de la obra de Antonio Pasquali. En: Bisbal, M. y Cañizález, A. (eds.). Travesía intelectual de Antonio Pasquali. A propósito de los 50 años de Comunicación y cultura de masas, pp. 51-79, Venezuela, UCAB/Konrad Adenauer.

ALTAMIRANO, C. (2005): Para un programa de historia intelectual y otros ensayos, Buenos Aires, Siglo XXI

Álvarez, A. y Rivera, M. (2011). Momentos iniciales: el Instituto de Arte. En: Kizer, G. (comp.) Retrospectiva de la Escuela de Artes: 1978-2008, Caracas, UCV.

ANZOLA, P. y COOPER, P. (1985): La investigación en comunicación social en Colombia, Lima, Desco.

BECERRA, M. (s/f): La sociedad de la información. Mimeo.

BECERRA, M. y MASTRINI, G. (2006): La economía política de la comunicación vista desde América Latina. En Revista E-Compós, Brasil, Diciembre. p. 2-20.

CAÑIZÁLEZ, A. (2014): El modelo de comunicación de Antonio Pasquali. En: Bisbal, M. y Cañizález, A. (eds.). Travesía intelectual de Antonio Pasquali. A propósito de los 50 años de Comunicación y cultura de masas, pp. 81-91, Venezuela, UCAB/Konrad Adenauer.

CAPRILES, O. (1976): El estado y los medios de comunicación en Venezuela, Caracas, ININCO.

(1979): Elementos para un análisis crítico del Nuevo Orden Internacional de la Comunicación y la Información, Caracas, UCV.

(1980): De las Políticas Nacionales de Comunicación al Nuevo Orden Internacional de la Información: algunas lecciones para la investigación. Ponencia presentada para la Conferencia Científica AIERI-IAMCR, Caracas, mimeo.

(1996): Poder político y comunicación, Caracas, UCV.

Chacón, A. (Ed.) (1971): La izquierda cultural, Caracas, Editora San José.

DELGADO FLORES, C. (2014): Al inicio de una ruta. Antonio Pasquali y la antropología de la comunicación. En: Bisbal, M. y Cañizález, A. (eds.). Travesía intelectual de Antonio Pasquali. A propósito de los 50 años de Comunicación y cultura de masas, 91-105, Venezuela, UCAB/Konrad Adenauer.

DOSSE, F. (2007): El arte de la biografía: entre historia y ficción, México, Universidad Iberoamericana.

ESTEINOU MADRID, J. (2014): El impulso de Antonio Pasquali al desarrollo de las Ciencias de la Comunicación en América Latina. En: Bisbal, M. y Cañizález, A. 
(eds.). Travesía intelectual de Antonio Pasquali. A propósito de los 50 años de Comunicación y cultura de masas, pp. 113-133, Venezuela, UCAB/Konrad Adenauer.

FUENTES NAVARRO, R. (1991): Un campo cargado de futuro. El estudio de la comunicación en América Latina, Guadalajara, ITESO.

GRAZIANO, M. (1988). Política o Ley: Debate sobre el Debate. En Espacios, Facultad de Filosofía y Letras, Universidad de Buenos Aires.

(1997). Guía teórica $1^{\circ}$ parte. Carrera de Ciencias de la Comunicación. Buenos Aires, Universidad de Buenos Aires, Facultad de Ciencias Sociales.

KANT, I. ([1781] 1984): Crítica de la razón pura, volumen II, Argentina, Ediciones ORBIS.

MARQUES DE MELO, J. (1987): “Teoría e investigación de la comunicación en América Latina. Balance preliminar de los últimos 25 años", en Estudios sobre las Culturas Contemporáneas, año/vol. I, número 002, Universidad de Colima, México, pp. 53:72.

MARTÍN-BARBERO, J. (1987): Procesos de comunicación y matrices de cultura, México, Ediciones G. Gili.

MASTRINI, G. (2011): “Medios públicos y derecho a la comunicación: una aproximación desde América Latina". En Lecciones del portal, Disponible en http://www.portalcomunicacion.com/lecciones.asp?aut=76

MUJICA, H. (2010) [1967]: El imperio de la noticia, Venezuela, AVN. (1973). Apuntes para una sociología de la comunicación venezolana. Caracas, UCV.

PASQUALI, A.: (1960): Antología de textos para la cátedra de Información Audiovisual, Caracas, UCV.

(1964a): Comunicación y cultura de masas, Caracas, EBUC.

(1964b): "La cultura de masas es dirigida en Venezuela por una oligarquía de la información", en Chacón, Alfredo (Editor), La izquierda cultural, Caracas, Editora San José, pp. 188-194.

(1967): El aparato singular: análisis de un día de TV en Caracas, Caracas, UCV.

(1973): Sociologia e Comunicação, Petrópolis, Editora Voces.

(1975): “Latin America: our message or theirs?", en Getting the message across: an inquiry into successes and failures of cross-cultural communication in the contemporary world, Paris, The UNESCO press, pp. 56-76

(1978): Comprender la comunicación, Caracas, Monte Ávila. 
([1986a] 1991): "Qué es una radiodifusión de servicio público", en Pasquali, Antonio, El orden reina. Escritos sobre comunicaciones, Venezuela, Monte Ávila Editores.

([1986b] 1991): “Comunicación y Cultura”, en Pasquali, Antonio, El orden reina. Escritos sobre comunicaciones, Venezuela, Monte Ávila Editores.

([1988] 1991): “Industrias culturales en América Latina”, en Pasquali, Antonio, El orden reina. Escritos sobre comunicaciones, Venezuela, Monte Ávila Editores.

en colaboración con Vargas, Armando (Editores), (1990), De la marginalidad al rescate: los servicios públicos de radiodifusión en la América Latina 1990, Costa Rica: Editorial Universidad Estatal a distancia.

(1990): La comunicación cercenada, Venezuela, Monte Ávila Editores.

(1991): El orden reina. Escritos sobre comunicaciones, Venezuela, Monte Ávila Editores.

(1998): Bienvenido Global Village, Venezuela, Monte Ávila Editores Latinoamericana.

PINEDA DE ALCÁZAR, M. (2010): Antonio Pasquali: la vigencia de su pensamiento cuarenta años después. En Chasqui. Revista Latinoamericana de Comunicación, n¹09, Quito, CIESPAL, pp. 18-20.

(2014): Antonio Pasquali: la vigencia de su pensamiento cincuenta años después. En: Bisbal, M. y Cañizález, A. (eds.). Travesía intelectual de Antonio Pasquali. A propósito de los 50 años de Comunicación y cultura de masas, pp. 21-31, Venezuela, UCAB/Konrad Adenauer.

RIVERA, J. (1987): La investigación en comunicación social en Argentina, Argentina, Puntosur.

SAFAR, E. (1978): La Información Audiovisual. Caracas. UCV.

(2014): Una constante en la obra de Antonio Pasquali: el Servicio Público de Radiotelevisión. En: Bisbal, M. y Cañizález, A. (eds.). Travesía intelectual de Antonio Pasquali. A propósito de los 50 años de Comunicación y cultura de masas, pp. 47-59, Venezuela, UCAB/Konrad Adenauer.

SAFAR, E. y PASQUALI, A. (1992): Memorias de un país en subasta I. La comunicación social, Mérida, Universidad de los Andes.

(1994): Memorias de un país en subasta II. Las telecomunicaciones, Mérida, Vértice Editores.

SAINTOUT, F. (2003) (editora): Abrir la comunicación. Tradición y movimiento en el campo académico, La Plata, Facultad de Periodismo y Comunicación Social (UNLP). 
SANCHEZ NARVARTE, R. E. (2016a): "El itinerario intelectual de Antonio Pasquali: aspectos teórico-metodológicos para pensar su abordaje", en Arias, Ana y López, Matías (Editores), Indisciplinas. Reflexiones sobre prácticas metodológicas en ciencias sociales, pp. 141-159, La Plata, Club Hem Editores.

(2016b): "Antonio Pasquali: apuntes para un itinerario intelectual", en Anuario de Investigaciones, vol. 2, pp. 141-172, La Plata, Facultad de Periodismo y Comunicación Social (UNLP).

SILVA, L. (2011) [1970]: La plusvalía ideológica, Venezuela, El Perro y la Rana.

TERÁN, O. (2008): Historia de las ideas en la Argentina. Diez lecciones iniciales, 18101980, Buenos Aires, Siglo Veintiuno Editores.

TORRES, F. y DE LOS REYES, D. (2009): Rompecabezas de una obra: Antonio Pasquali y su utopía comunicacional, Caracas, UCAB.

TREJO DELARBRE, R. (2014): Antonio Pasquali: Cátedra Social, Ejemplo Público. En: Bisbal, M. y Cañizález, A. (eds.). Travesía intelectual de Antonio Pasquali. A propósito de los 50 años de Comunicación y cultura de masas, pp. 71-81, Venezuela, UCAB/Konrad Adenauer.

WILLIAMS, R. (2009) [1977]: Marxismo y literatura, Argentina, Las Cuarenta.

ZAROWSKY, M. (2013): Del laboratorio chileno a la comunicación-mundo. Un itinerario intelectual de Armand Mattelart, Buenos Aires, Biblos. 